\title{
Qualitative Interviews of Romanian Key Informants Guiding a Preliminary Health Needs Assessment of Romanian Immigrants in Southern California
}

\author{
Tabitha D. Dutciuc ${ }^{1,2}$, Nicholas Gorman ${ }^{1}$, Sora Park Tanjasiri ${ }^{1}$, and Brandon Brown ${ }^{2}$ \\ ${ }^{1}$ California State University, Fullerton \\ ${ }^{2}$ University of California, Irvine
}

\begin{abstract}
Background: Current literature suggests that immigrant populations face greater challenges in regards to health and healthcare which are caused by a variety of factors, including language, socioeconomic status, acculturation, and health beliefs. The scope of this research was to conduct a preliminary health needs assessment of the Romanian-American population as a first step towards creating culturally tailored health programs to meet their needs. Methods and Results: Qualitative data was acquired through semistructured interviews with key stakeholders $(n=5)$ in the Romanian health care system, in Romania, revealing a total of 5 themes. The data was compiled and analyzed to discover the main health beliefs that contribute to the health behaviors present in this population. Conclusion: These data begin to identify important health beliefs and risk factors unique to this population and clarify priorities for future research in the U.S.
\end{abstract}

(C) 2014 Californian Journal of Health Promotion. All rights reserved.

Keywords: Romanian, immigrant health, qualitative, needs assessment

\section{Introduction}

Some of the most pronounced health-related disparities exist among immigrant populations. Current literature suggests that immigrant populations face greater challenges in regards to access to healthcare and health outcomes, both of which are influenced by a variety of factors, including language, socioeconomic status, acculturation, and health beliefs (Argeseanu Cunningham, Ruben, \& Venkat Narayan, 2008; Angel, Buckley, \& Sakamoto, 2001).

\section{Health Disparities among White Immigrants}

While the health risks and disparities faced by these populations are generally well documented, there exist subpopulations whose risk profiles remain understudied. This may be due, in part, to the fact that some high-risk populations become aggregated into the broad category of "White/Caucasian" thus obscuring their unique health profile. For example, when looking at epidemiological literature specific to Eastern European health, there have been a number of serious health risks and disproportionate mortality patterns compared to other "White/Caucasian" populations that have been documented (Nasseri, 2008; Hofmann, 2012). In another example, researchers found that Romania has the highest cervical cancer incidence and mortality rates in the European Union, indicating lower rates of screening and HPV vaccination behaviors, despite HPV immunization campaigns launched by the Romanian Ministry of Health in 2008 and 2009 throughout the country (Craciun \& Baban, 2012; World Health Organization, 2010). By aggregating these white immigrant populations under the broad racial category of "White/Caucasian" in larger epidemiological studies there runs the risk of obfuscating the unique and substantive health risks faced by these immigrant populations from different countries of origin. As a result, there remains a need for further research in this vein.

Though studies of Eastern Europeans are illustrative, evidence suggests that phenomenon may be present in southern California. For instance, a study of mortality patterns in first generation non-Hispanic White immigrants of southern California found higher mortality rates linked to behavior (chronic diseases that begin early in life and are acquiescent to early 
detection, such as diseases of the heart, diabetes, and certain cancers) and social environments (suicide) (Nasseri, 2008). Culture-specific health beliefs among White immigrants, such as a paternalistic relationship with providers and behaviors such as medical check-ups and healthy eating affect attitudes toward preventative care and possibly subsequent preventative behaviors and treatment.

\section{Romanian Immigrants}

One subpopulation of concern is Romanian immigrants. The United States Census Bureau reports that nearly 69,000 persons of Romanian descent reside in California (US Census Bureau, 2013). The Centers for Disease Control and Prevention reports that across several counties in southern California, Romanian ranks among the top 20 in languages spoken at home (CDC, 2010). With large concentrations of Romanian immigrants residing in southern California, there is both a need as well as an opportunity to study and analyze the health disparities facing this cultural group. In many cases, medical providers are not able to effectively communicate the severity of diseases to immigrant patients and consequently are unable to create a plan of health or prevention strategies for immigrant patients (Shaw, Huebner, Arminm, Orzech, \& Vivian, 2009). Since Romanian immigrants often exemplify an attitude of passiveness towards health care, it is necessary to assess the needs of this cultural group and develop a strategy for developing culturally tailored programs and using gatekeepers in addressing the needs of this population (Todorova et al., 2009). The purpose of this research project was to perform a preliminary health needs assessment of Romanians, in order to create culturally tailored health programs for Romanian immigrants in Southern California.

\section{Participants}

\section{Methods}

During July-August 2009, the primary author traveled to Romania to conduct semi-structured interviews with five key informants who lived in Romania. A 30-minute recorded interview was conducted with two physicians from a large city in North-Eastern Romania (a general practitioner and her daughter, who is a specialist physician) and shorter, unrecorded interviews with three other health professionals (a general surgeon, a laboratory technician, and a pediatrician) from a large city in Western Romania.

\section{Procedures}

The participants provided written and verbal consent to being recorded and used as part of the research project. Due to the logistic constraints of the trip, a convenience sampling approach was taken in the selection of participants. The aforementioned physicians were selected on the basis of their availability and willingness to participate in the study and were recruited based on introductions through acquaintances and professional recommendations. Inclusion criteria were that the participants were health professionals who were currently working in the Romanian healthcare sector and were willing to participate in semi structured, "conversationtype" interviews. Participants were excluded if they were not willing to participate and were not actively employed health professionals. Participants who preferred to not be recorded were included if they consented to having a conversation during which the researcher took notes.

\section{Human Subject Approval}

In order to ensure ethical data collection and key informant interviews with human subjects, the research instruments and protocol were reviewed and approved the California State University, Fullerton Institutional Review Board.

\section{Measures}

During these interviews, a special focus was placed on the topics of health promotion and disease prevention. The physicians were asked questions regarding the health care system in Romania, the health status of Romanians, main causes for morbidity in Romania, the relevant health beliefs and behaviors of Romanians, and their views on the characteristics of the Romanian lifestyle that contribute to disease. Informal interviews with the three additional health professionals gave insight into the aspects of Romanian culture that may pertain to health beliefs and outcomes of Romanians in southern California. These data were compared with 
unpublished results from a quantitative survey pilot of Romanian Americans.

\section{Table 1.}

\section{Semi-structured Interview Emergent Themes}

\section{Gaps in the Healthcare System}

"All patients have a family doctor that they must go through: the family doctor who is familiar with the patient's history first before receiving any other specialized care" Specialist, Romania

"I also think that there is a lack of a good scheduling system here in Romania. The Romanians is not obligated by anyone to get their colonoscopy, pap test, mammography...if he or she wants to, they are provided for free, but no one is pushing the citizens to get these tests." -Specialist, Romania "Also, there is a lack of consistent funding from the state. Often times at a laboratory, for example, if a patient goes to get a test, the staff may say 'we don't have any money allocated from the health insurance, so you have to pay'. This also discourages people from getting tests and screenings."

-Specialist, Romania

Preventative Care

"There is of course, the classic Romanian behavior of not visiting the medic unless they are in unbearable pain” Specialist, Romania

"In terms of preventative care, it depends on the patient and the doctor. If the patient seems interested, the doctor will tell him what to do." -General Practitioner, Romania "I think that the physicians are fairly conscientious and do their duty of sending their patients to get preventative care." -Specialist, Romania

"The doctor send the patient [to get screenings and preventative care], it depends on the patient [whether or not they go]"-General Practitioner, Romania

Health Beliefs/Attitudes

"Just yesterday I went to the store and observed a woman ask for a pack of cigarettes. She told the clerk not to give her the [package labeled] with the cancer [warning], but instead the other package with the less provocative message because it was the 'weaker' cigarette.”-Specialist, Romania

Health Behaviors

"Romania is becoming more westernized... so many of the [negative] things from the west are infiltrating the Romanian culture. For example, many people have started consuming more fast food, and [have] a more stressful lifestyle... But even so, the traditional Romanian foods are not exactly 'healthy' either... sarmale [cabbage rolls with meat-usually pork-and rice as the main ingredients], fried meat, etc... and that is why I believe we see so many cancers"

-Specialist, Romania

"We now know that breast cancer is associated with the used of contraceptives, cervical cancer is caused by the number of sexual partners and the human papillomavirus, colon cancer is caused by diet, and lung cancer by smoking." -Specialist, Romania

Stigmatized Diseases

"I don't think that people avoid seeking medical help if they might have a stigmatized disease, because people know that the moment that you come out with the disease, there is treatment available to help you” -Specialist, Romania

\section{Data Management and Analyses}

The two "formal" interviews were audio recorded and transcribed verbatim. Informal interviews took the format of a semi-structured conversation wherein the researcher asked questions or proposed topics to which the key informants provided open-ended feedback. The interviewer took notes from the three "informal" unrecorded interviews and developed a table of themes that were identified based on the question categories derived from literature reviews and researcher observations of health in Romania. The interviews were unrecorded and "informal" to provide a less structured, conversational approach to identifying the recurring themes and key informant opinions. The primary author applied systematic comparative and descriptive content analysis that consisted of grouping and coding the transcribed information in thematic categories, then identifying recurring issues and differences in the narratives and researcher notes through qualitative inductive analysis (Patton, 2002). A second reviewer confirmed the analysis and discrepancies were resolved. Representative quotes were extracted and translated into English. Five thematic categories were identified and the representative quotes were organized in a table.

\section{Results}

Thematic analysis of the formal interviews yielded several emergent themes (See Table 1 for a review and representative quotes). A total of five thematic categories emerged from the qualitative analysis: health behaviors, health beliefs/attitudes, Romanian health promotion, gaps in the Romanian healthcare system, and stigmatized diseases. The physicians provided insight into the general population by discussing their own experiences with patients and mentioned respiratory disease, cardiovascular disease, and cancers (for men: lung, prostate, and colorectal, and for females: breast, cervical, and colorectal) as the primary patient morbidities encountered in their practice.

\section{Health Behaviors}

Over the years, incidence rates for the top three morbidities (respiratory disease, cardiovascular 
disease, and cancers) have increased because "Romania is becoming more westernized... so many of the [negative] things from the west are infiltrating the Romanian culture. For example, many people have started consuming more fast food, and [have] a more stressful lifestyle. But even so, the traditional Romanian foods are not exactly 'healthy' either... sarmale [cabbage rolls with meat - usually pork - and rice as the main ingredients], fried meat, etc... and that is why I believe we see so many cancers" (Personal communication, July 16, 2009). Another disease that the physicians discussed in depth was respiratory disease-caused by the high prevalence of smoking in Romania. Second hand smoke has also lead to increased rates of asthma and upper respiratory problems among nonsmokers.

\section{Health Beliefs/Attitudes}

Though many of the leading causes of morbidity among Romanians mirror those of the greater Caucasian population, interviews emphasized the importance of considering Romanians' health attitudes. Health attitudes, such as distrust of the Romanian health care system, were another theme the doctors touched upon in the interviews. The importance of looking at health attitudes such as distrust of the healthcare system is underscored by reports of Romanian's underutilization of screening proceduresespecially of cervical screenings.

The historical context of the transitional health system of Romania emerged as a theme in the informal interviews, where it was suggested that paternalistic preventative messages are often a barrier to seeking care. During the years of the communist government regime, Romanians were pressured to receive mandatory preventative care in order to achieve an ideal population health status. As a result, dictated health prevention messages may not be received well in the general population, as they are a reminder of the "previous system's” agenda.

The key informants also provided an excellent source of information on the behavioral characteristics of Romanians that may lead to increased risk for diseases. "There is of course, the classic Romanian behavior of not visiting the medic unless they are in unbearable pain," which often results when the problem has aggravated to a more severe state. Consequently, the tendency to disregard screenings often leads to late diagnosis and lower rates of receiving preventative care, which, according to the two physicians, also increases risk for morbidity in the population as a whole. These health behaviors and beliefs were also evident in the researcher's observations in Romania and among Romanian-Americans in southern California.

\section{Romanian Health Promotion}

One of the most visible aspects of health promotion in Romanian society is the use of mass media (television and radio) as a facilitator of public health service announcements. Romanian TV airs anti-smoking and "healthytips" commercials several times each hour to remind viewers to practice healthier lifestyles, to educate them about the benefits of living healthier, and to warn them of the consequences of poor health behaviors. Though there is information available to the Romanians, individuals seem to ignore it. The younger physician informant recalled an example of this phenomenon: "Just yesterday I went to the store and observed a woman ask for a pack of cigarettes. She told the clerk not to give her the [package labeled] with the cancer [warning], but instead the other package with the less provocative message because it was the 'weaker' cigarette.” Though warning labels are placed on every carton of cigarettes, and health messages are promoted through mass media, smoking is allowed in malls, bars, and almost every public place. Likewise, fast food restaurants are located in malls, town squares, and at almost any social meeting place of major cities. It is not uncommon to see American fast food chains, located in historical places or downtown areas, such as the famous Piața Victoriei (Victory Square) in downtown Timişoara.

It is also worth noting that while positive health messages abound, several important sources of negative behavior also exist. For instance, the messages conveyed by health workers both verbally and through their actions tell a story in 
contrast to that of anti-smoking materials. The researcher conducted informal interviews with two other health professionals (one of whom was a general surgeon at a county hospital in a major Romanian city, and the other a laboratory technician from a children's hospital) who were smokers that were not willing, nor saw it necessary, to change their behaviors.

\section{Gaps in the Romanian Health Care System}

All participants also mentioned that there were significant gaps the in the Romanian health care system. It is often difficult to encourage preventative care because although Romania has a universal healthcare system, funding for certain tests and procedures may not be allocated. Also, many preventative procedures, such as cervical cancer screenings and vaccinations, must be conducted in specialist offices, thus requiring patients to schedule additional appointments. There are gaps in patient surveillance for scheduling and organization to require participation in preventative services. A physician may base his/her prescription for preventative care on how willing and amiable the patient is.

\section{Stigmatized Diseases}

Interview questions were also tailored to probe for information regarding any potentially stigmatized diseases in the Romanian culture. While several illnesses were identified, mental illness was discussed as being the most stigmatized among the Romanian culture. "Of course parents always want to make sure that their children don't marry someone [with mental illness] in their family... we know [lots of mental illnesses] are genetic", said one physician. In terms of other stigmatized diseases or conditions, physicians agreed that Romanians tend to accept physical diseases, such as HIV/AIDS quicker than they would mental illness, as there is treatment available to address physical symptoms. Because mental illness is perceived to be incurable or generally more difficult to treat, it remains to be greatly stigmatized.

\section{Discussion}

\section{Historical Context}

The results of the present study were largely in line with the existing literature on immigrant health and illustrate the need for additional research into the unique risks faced by Romanian Americans. Results from the qualitative interviews indicated that Romanians might be resistant to some health promotion efforts due to a paternalistic historical context and a passive attitude toward seeking preventive care. The emergent themes from the qualitative data suggest lingering implications from years of communist oppression and a transitional situation in Eastern Europe and Romania. These effects lead to social discourse on disease prevention and health promotion efforts (Todorova et al., 2006). Research suggests that, in former communist countries, the tendency to implement a free market economy in the health sector is a reaction to decades of centralism and authoritarianism from the communist period often resulting in fragmentation of promotion and preventative services and an underdevelopment of the different levels of care (Vlădescu, et al., 2008). The health attitudes that can be carried over during the immigration process can linger, and call for further evaluation of the health beliefs and attitudes that Romanians in the U.S. have. Understanding the historical context can serve as a gateway for creating culturally appropriate programs that focus on health programs through communitybased interventions rather than through policy changes. Further insight is needed to measure the impact of the historical context among Romanians immigrants (e.g., in southern California) as a way of measuring acculturation, as well as health attitudes and beliefs.

\section{Preventative Care}

The health beliefs and behaviors derived from these transitional and oppressive historical contexts are paralleled with the health disparities and epidemiological data in literature (WHO, 2012; Vlădescu, et al., 2008). With less regulation from the government, Romanians will tend to neglect the screenings and health promotion efforts that were once mandated 
(Todorova, et al., 2006). The high incidence and mortality of diseases such as cervical cancer is an example of the lingering implications of these situations that can follow an immigrant to the new country of residence. This is especially significant, as many public health reforms and evidence-based health promotion strategies have been implemented as recently as 2004-long after many immigrant groups have transitioned to new countries during and immediately after the period of Communism in the late 1980's and early 1990's (Vlădescu, et al., 2008). The health attitudes and behaviors that were present at the time of immigration may remain with immigrants through the acculturation process. Further qualitative research is needed to assess the attitudes and behaviors associated with preventative care among Romanians in southern California. Other studies have demonstrated that immigrant groups from the former Soviet Union experience a frustration with the U.S. Health Care System due to the difficulty of navigating through the system and the differences from that of their native country (Van Son \& Gileff, 2013). If the Romanian-American population experiences similar frustrations, interventions can be tailored to community groups in promoting preventative care in a culturally competent manner and assisting immigrants with seeking preventative care.

\section{Social Support}

The qualitative findings regarding the stigmatization of mental illnesses are also of concern given research suggesting that immigrant populations have higher rates of mental health issues and stress (Nasseri, 2008; Arcia, Skinner, Baily, Correa, 2001; Leclere, Jensen, Biddlecom, 1994). Social support is often an important indicator of mental health and merits further study. In an unpublished pilot survey comparing Romanian-American churchgoers in southern California to the nonHispanic White population, $44.4 \%$ of individuals said that they "sometimes, rarely, or never" received the social support they needed (compared to the $18.2 \%$ of the general white population who gave those answers). Results were significantly lower for responding to "always" receiving social support compared to the NHW population (33.3 \% for Romanian-
American sample versus $47.0 \%$ general population) (unpublished results).

However, in other studies assessing social support among immigrant groups in California, the tight social networks often serve as excellent sources of high levels of social support regardless of their impact on health (Finch \& Vega, 2003). Other European immigrant groups also tend to report higher levels of social support, which may aid in navigating through the new country's health care system and in accelerating acculturation (Van Son \& Galiff, 2013; Jasinskaja-Lahti, Liebkind, Jaakkola, \& Reuter, 2006). The differences in availability of social support in the Romanian-American population compared to the general white population, as well as the differences of this population compared to other immigrant groups from Europe and within California, are significant and merit further investigation, especially in the context of health. The insight gleaned from the qualitative findings also calls for further conversations and investigations with the Romanian-American population to identify factors that impact social support and perceptions on mental illness.

\section{Delaying Medical Care}

Delaying needed medical care is also of particular concern for this population. The abovementioned pilot study also showed a statistically significant delay in getting needed medical attention in the Romanian-American churchgoer sample (44.4\% versus $14.6 \%$ in the general population) (unpublished results). Historically, most immigrants have tended to be self-employed and/or have worked for employers who could not afford health insurance, therefore relying on safety net hospitals and clinics and delaying care (Light, 2011). It is worth noting that access to healthcare may not be the only contributing factor to delaying medical care, but may also be deeply rooted within attitudes and behaviors carried over from Romania. In fact, the issue of delaying medical care emerged in the qualitative interviews and has been demonstrated in the health statistics from Romania. For example, the World Health Organization (WHO) reports that Romania has the highest incidence and mortality 
of cervical cancer in the European Union (WHO, 2010), a finding partially attributable to the low levels of screenings observed in this population (Arbyn, Raifu, Weiderpass, Bray, \& Antilla, 2009). As a disease often characterized by morbidity brought on by disparity and best addressed through early detection, screening, risk factor reduction, and vaccination (Kesic, Poliak, \& Rogoskava, 2012), cervical cancer serves as an illustrative example of the significance and cost of delaying medical procedures. As a result, the delaying behaviors observed here, if borne out in the Romanian immigrant population in California, would be worth further qualitative and quantitative examination, with an emphasis on preventable disorders like some cancers and those most aggravated by delaying medical care.

\section{Limitations}

It should be noted that there were several limitations to the current study. Specifically, sample size impacts the generalizability of the findings. As the qualitative materials that were gathered were drawn from five key informants in the country of Romania, they may not best represent the health needs of Romanians in America or the opinions of the broader community of health practitioners in Romania. While sample size considerations in qualitative research are typically determined by saturation, this project, as an unfunded pilot study was limited in duration, allowing for the interview of only five subjects. Further research is needed to establish additional or alternative themes, as the present sample was limited.

\section{Conclusion}

Our qualitative data shows that Romanians have unique health behaviors and beliefs that set them in a significantly higher risk group than the broader "white" category might convey. Given the relatively large population of Romanian immigrants living in southern California, future research into the health behavior/beliefs of this population and the impact of acculturation on its members is warranted. Recommendations for the continuation of research include conducting a rigorous quantitative component from an organizational sample of "Romanian" affiliated church denominations as well as a "nonreligious" domain of Romanian-Americans. There is a need to further develop questionnaire materials and develop items that will address the scope of the needs assessment in a more direct way. This preliminary health needs assessment serves as a map for additional research among Romanian-American health indicators, including those regarding cervical cancer in RomanianAmerican women. Though it may be premature to make policy recommendations for this ethnic group, results from the present study are suggestive; the possibility of unique health beliefs, risks, and needs within the RomanianAmerican population merits further investigation.

\section{Acknowledgements}

The authors would like to acknowledge the following individuals for their contribution to the research and honors project: Dr. Susan Jacobsen, director of the California State University, Fullerton Honors Program; Alina Sarbu, Adrian Haj, and Alina Maciujec, for their hard work and contribution to the translation process; and Ms. Sandra Perez for administrative support in the manuscript preparation.

\section{References}

Angel, J. l., Buckley, C. J., \& Sakamoto, A. (2001). Duration or disadvantage? Exploring nativity, ethnicity, and health in midlife. Journal of Gerontology: Social Sciences, 56B(5), S275-S284.

Arbyn M., Raifu, A.O., Weilderpass, E., Bray, F., \& Anttila, A. (2009). Trends of cervical cancer mortality in the member states of the European Union. European Journal of Cancer, 45(15), 2640-2648 [special issue].

Arcia E., Skinner M., Baily D., \& Correa V. (2001). Models of acculturation and health behaviors among Latino immigrants in the U.S. Social Science Medicine, 53(1), 41-53. 
Argeseanu Cunningham, S., Ruben, J. D., \& Venkat Narayan, K. M. (2008). Health of foreign-born people in the United States: A review. Health \& Place, 14, 623-635.

Centers for Disease Control and Prevention. (2007). Emergency preparedness and response: Riverside County, California languages spoken at home detail, 2007. Available at http://emergency.cdc.gov/snaps/data/06/06065_lang.htm. Accessed January 16, 2013.

Centers for Disease Control and Prevention. (2007). Emergency preparedness and response: Orange County, California languages spoken at home detail, 2007. Available at http://emergency.cdc.gov/ snaps/data/06/06059 lang.htm. Accessed January 16, 2013.

Craciun, C. \& Baban, A. (2012). Who will take the blame? Understanding the reasons why Romanian mothers decline HPV vaccination for their daughters. Vaccine, 30, 6789-6793.

Finch, B. K., \& Vega, W. A. (2003). Acculturation stress, social support, and self-related health among Latinos in California. Journal of Immigrant Health, 5(3), 109-117.

Hofmann, E.T. (2012). The burden of culture? Health outcomes among immigrants from the Former Soviet Union in the United States. Journal of Immigrant \& Minority Health, 14(2), 315-322.

Jasinskaja-Lahti, I., Liebkind, K., Jaakkola, M., \& Reuter, A. (2006). Perceived discrimination, social support networks, and psychological well-being among three immigrant groups. Journal of Cross-Cultural Psychology, 37(3), 293-311.

Kesic, V., Poliak, M., Rogovskaya, S. (2012) Cervical cancer burden prevention activities in Europe. Cancer Epidemiology, Biomarkers, \& Prevention, 21, 1423-1433.

Leclere F.B., Jensen L., \& Biddlecom A.E. (1994). Health care utilization, family context, and adaptation among immigrants to the United States. Journal of Health and Social Behavior, 35, 370-384.

Light, D. W. (2011). Categorical inequality, institutional ambivalence, and permanently failing institutions: the case of immigrants and barriers to health care in America. Ethnic and Racial Studies, 35(1), 23-39

Nasseri K. (2008). Mortality in first generation white immigrants in California, 1989-1999. Journal of Immigrant and Minority Health, 10(3),197-205.

Patton, M.Q. (2002). Qualitative research and evaluation methods. Thousand Oaks, CA: Sage.

Shaw S.J., Huebner C., Armin J., Orzech K., \& Vivian J. The role of culture in health literacy and chronic disease screening and management. Journal of Immigrant Minority Health, 11(6), 460-7. doi: 10.1007/s10903-008-9135-5

Todorova, I., Baban, A., Alexandrova-Karamanova, A., \& Bradley, J. (2009). Inequalities in cervical cancer screening in Eastern Europe: perspectives from Bulgaria and Romania. International Journal of Public Health, 54, 222-232.

Todorova, I. L. G., Baban, A., Balabanova, D., Panayotova, Y., \& Bradley, J. (2006). Providers' constructions of the role of women in cervical cancer screening in Bulgaria and Romania. Social Science \& Medicine, 63(3), 776-87. doi:10.1016/j.socscimed.2006.01.032

U.S. Census Bureau, 2009-2011 American Community Survey. Retrieved July 1, 2013, from http://factfinder2.census. gov/faces/tableservices/jsf/pages/productview.xhtml?pid=ACS_10_SF4_DP02\&prodType=table

Van Son, C. R., \& Gileff, T. Y. (2013). Relying on what they know: older Slavic emigres managing chronic health conditions. Qualitative Health Research, 23(12), 1660-71. doi:10.1177/ 1049832313508842

Vlădescu, .C, Scîntee, G., Olsavszky, V., Allin, S., \& Mladovsky, P. (2008). Romania: Health system review. Health Systems in Transition, 10(3), 1-172.

World Health Organization. (2012). Evaluation of structure and provision of primary care in Romania: A survey-based project. Available at http://www.euro. who.int/ data/assets/pdf file/0005/167576/Evaluation-of-structure-and-provision-of-primarycare-in-Romania.pdf. Accessed March 3, 2013.

WHO/ICO Information Centre on HPV and Cervical Cancer (HPV Information Centre). (2010). Human Papillomavirus and related cancers in world: Summary report 2010. Available at http://apps.who.int/hpvcentre/statistics/dynamic/ico/country_pdf/XWX.pdf?CFID=7177599\&CF 
TOKEN=13866214. Accessed January, 16, 2013.

Author Information

Tabitha D. Dutciuc, MPH

California State University, Fullerton/University of

California, Irvine

Program in Public Health

Nicholas Gorman, MPH, EdD

California State University, Fullerton

School of Nursing

Sora Park Tanjasiri, MPH, DrPH

California State University, Fullerton

Department of Health Science

*Brandon Brown, MPH, PhD

University of California, Irvine

Program in Public Health

Department of Population Health \&

Disease Prevention

653 E. Peltason Dr., AIRB Rm. 2024

Irvine, CA 92697-3957

brandon.brown@uci.edu

(949) 824-6996

(949) 824-0529 fax

* corresponding author 